\title{
ON THE NUMBER OF AUTOMORPHISMS OF A CLOSED RIEMANN SURFACE
}

\author{
BY \\ ROBERT D. M. ACCOLA $\left({ }^{1}\right)$
}

1. Introduction. For an integer $g$ greater than or equal to 2 , let $N(g)$ be the order of the largest group of conformal self-maps (automorphisms) that a Riemann surface of genus $g$ can admit. A. Hurwitz [3] proved that $N(g) \leqq 84(g-1)$. A. M. Macbeath [4] proved that $N(g)=84(g-1)$ for an infinite number of integers $g$. Macbeath [5] also proved that $N(g)<84(g-1)$ for an infinite number of $g$ 's $\left({ }^{2}\right)$. The purpose of this paper is to prove that $N(g) \geqq 8(g+1)$, and this lower bound is sharp for an infinite number of $g$ 's. The methods used show also that if $g$ is divisible by 3 then $N(g) \geqq 8(g+3)$, and again the bound is attained infinitely often. (The same results have been obtained independently by Colin Maclachlan [6].)

To prove the weak inequality in the principal result, we construct for each $g$ a surface admitting a group of order $8(g+1)$. A slightly more complicated construction is needed for the $8(g+3)$ case. The constructions in $\$ 2$ follow well-known procedures. To prove the sharpness of the bounds for certain $g$ we eliminate the possible larger groups. This is more difficult than the constructions and is accomplished in $\$ 4$. Unfortunately, no general principle seems to be in force. Different kinds of groups must be eliminated by different methods. $\$ 3$ contains some group theoretic results needed in $\$ 4$.

The approach to the subject of automorphisms in this paper is equivalent to that of A. M. Macbeath [4], [5], although it is technically a little different. The approach is that of Seifert and Threlfall [8] and H. Röhrl [7]. All these methods, of course, go back to Hurwitz [3].

Suppose that $W$ is an $n$-sheeted ramified covering of $W_{0}$ and that $f$ is the covering map. We will always assume that the covering is unbounded; that is, any path $\gamma_{0}$ in $W_{0}$ can be continued from any initial point in $W$ lying over the initial point of $\gamma_{0}$. Since $W$ is ramified over $W_{0}$ continuations need not be unique. Let $T_{0}$ be the set of points of $W_{0}$ over which $W$ is branched. Let $W_{0}^{\prime}=W_{0}-T_{0}$ and let $W^{\prime}$ $=W-f^{-1}\left(T_{0}\right)$. Then $W^{\prime}$ is a smooth unbounded covering of $W_{0}^{\prime}$ and continuations here are unique. Let $F_{0}$ be the fundamental group of $W_{0}^{\prime}$ based at $p_{0}$. Let $\left\{p_{1}, \ldots, p_{n}\right\}$ $=f^{-1}\left(p_{0}\right)$. If $\gamma_{0}$ is a path in $W_{0}^{\prime}$ beginning and ending at $p_{0}$, let $\gamma_{i}$ be the lift of $\gamma_{0}$ starting at $p_{i}, i=1,2, \ldots, n$. The permutation of $\left\{p_{1}, \ldots, p_{n}\right\}$ given by sending the

Presented to the Society, March 5, 1965 under the title On automorphisms of compact Riemann surfaces; received by the editors January 23, 1967.

( $\left.{ }^{1}\right)$ Research supported by the Office of Naval Research.

$\left(^{2}\right)$ This last result of Macbeath will be an incidental consequence of the work of this paper. 
initial point of $\gamma_{i}$ into its terminal point (for each $i$ ) depends only on the homotopy class of $\gamma_{0}$. Thus to each element of $F_{0}$ is associated a permutation in $\Sigma_{n}$, the symmetric group on $n$ objects. This association is a homomorphism (or antihomomorphism, depending on how one writes his permutations) and will be denoted by $\mu$. The covering $W^{\prime} \rightarrow W_{0}^{\prime}$ is uniquely determined by $\mu$. Conversely, the covering $W^{\prime} \rightarrow W_{0}^{\prime}$ determines $\mu$ up to conjugacy; that is, up to a renumbering of the points of $f^{-1}\left(p_{0}\right)$. Moreover, the branched covering $W \rightarrow W_{0}$ is also uniquely determined by $\mu$, for given the covering $W^{\prime} \rightarrow W_{0}^{\prime}$, there is only one way to extend it to $W$ to be continuous at the isolated points of $f^{-1}\left(T_{0}\right)$. The image $\mu\left(F_{0}\right)$ is a transitive subgroup of $\Sigma_{n}$.

The relevence of this approach to the subject of this paper is contained in the equivalence of the following five conditions:

(1) The order of $\mu\left(F_{0}\right)$ is $n$.

(2) $\mu\left(F_{0}\right)$ is a regular subgroup of $\Sigma_{n}$.

(3) The kernel of $\mu, F$, can be identified with the fundamental group of $W^{\prime}$.

(4) $W^{\prime}$ admits a group, $G^{\prime}$, of automorphisms of order $n$ so that $W_{0}^{\prime}=W^{\prime} / G^{\prime}$.

(5) $W$ admits a group, $G$, of automorphisms of order $n$ so that $W_{0}=W / G$.

Under these conditions the elements of $G^{\prime}$ are the restrictions to $W^{\prime}$ of the elements of $G$ and $\mu\left(F_{0}\right)$ is isomorphic to $G$.

In this paragraph assume that one (and therefore all) of the above conditions hold for the covering. If $W_{0}$ has an automorphism $\phi_{0}$, we can lift $\phi_{0}$ to a $\phi$ on $W$ so that $f \circ \phi=\phi_{0} \circ f$ if and only if (a) $\phi_{0}$ permutes the points of $T_{0}$; that is, $\phi_{0}$ restricted to $W_{0}^{\prime}$ is an automorphism; and (b) $\phi_{0}$ acting on $F_{0}$ (in the natural way) maps the kernel, $F$, of $\mu$ onto itself. The action of $\phi_{0}$ on $F$ is well defined since $F$ is a normal subgroup of $F_{0}$. Consequently, if $W_{0}$ admits a group of order $m$ satisfying conditions (a) and (b) and if the order of $G$ is $n$, then $W$ will admit a group of automorphisms of order $m n\left(^{3}\right)$.

2. The construction of "big" groups of automorphisms. If $G$ is a finite group, denote the order of $G$ by $o(G)$. If the genus, $g$, of a surface is greater than 1, call a group, $G$, of automorphisms big if $o(G) \geqq 8(g+1)$.

Let $G^{\prime}$ be a given finite group of order $n$, which we may assume is a regular transitive subgroup of the symmetric group on the first $n$ integers, $\Sigma_{n}$. Let $W_{0}$ be a compact Riemann surface and let $W_{0}^{\prime}$ be $W_{0}$ punctured a finite number of times (possibly zero times). One finds a representation of $F_{0}$ into $\Sigma_{n}$ whose image is $G^{\prime}$. Then $W^{\prime}$, and so $W$, corresponding to this representation has a group $G$ isomorphic to $G^{\prime}$ so that $W_{0}=W / G$. Since the branching of $W \rightarrow W_{0}$ at a point $p^{\prime}$ of $W_{0}$ is determined by the order of $\mu\left(\gamma_{0}\right), \gamma_{0} \in F_{0}, \gamma_{0}$ "circles" $p$ ', the Riemann-Hurwitz formula [3] can be used to give the genus of $W$.

We now construct some big groups. Let $W_{0}$ be the Riemann sphere and let $W_{0}^{\prime}$ be $W_{0}$ punctured at the eight fixed points of order three of the octahedral group

$\left({ }^{3}\right)$ The material in this paragraph is a paraphrase of Macbeath's idea in [4]. 
$\left(\Sigma_{4}\right)$ [1]. The octahedral group, acting as a permutation group on these eight points divides the points into two sets of imprimitivity of four points each. Let $G^{\prime}$ be a cyclic group of order $n$, a transitive regular subgroup of $\Sigma_{n}$ with a generator $\phi$. If $F_{0}$ is the fundamental group of the eight-fold punctured sphere, define $\mu$ to be $\phi$ on the four paths going around the points of one set of imprimitivity and define $\mu$ to be $\phi^{-1}$ on the other four paths. $\mu$ is easily seen to be a representation and, moreover, it is not hard to see that any element of the octahedral group maps the kernel of $\mu$ into itself. Consequently, the surface $W$ corresponding to $\mu$ has a group of $24 n$ automorphisms. The covering $W \rightarrow W_{0}$ is branched only over the eight distinguished points and at each such point all the sheets come together in one point. Thus the total ramification of the covering $W \rightarrow W_{0}$ is $8(n-1)$. The RiemannHurwitz formula gives $2 g-2=n(-2)+8(n-1)$, or $g=3 n-3$. Consequently, $W$ admits a group of order $8(g+3)$. Since the construction does not depend on $n$, it follows that $N(g) \geqq 8(g+3)$ if 3 divides $g\left({ }^{4}\right)$.

To show that $N(g) \geqq 8(g+1)$ one merely constructs the hyperelliptic surface over the Riemann sphere with branch points of order 2 over each of the vertices of the regular $(2 g+2)$-gon inscribed in the unit circle. The dihedral group of order $4 g+4$ can be lifted which together with the interchange of the sheets gives a group of order $8(g+1)$ on the hyperelliptic surface $\left({ }^{5}\right)$.

3. Group theoretic preliminaries $\left({ }^{6}\right)$. In this section we gather together some group theoretic results needed in $\S 4$. The results are all elementary. The first three lemmas are well known [2], [9] and are stated without proof.

LEMMA 1. Let $S$ be a normal subgroup of a finite group $G$. The elements of $G$ are mapped into the automorphism group of $S, A(S)$, by considering $G$ as its set of inner automorphisms. If $S$ is abelian, the kernel of the map $G \rightarrow A(S)$ is a normal subgroup in which $S$ is central.

LEMMA 2. If $S$ has prime order $p$, then $o(A(S))=p-1$.

LEMMA 3. If a Sylow subgroup of a finite group, $G$, is central, then it has a normal complement and so $G$ is a direct product.

The remaining lemmas of this section concern special finite groups which arise naturally in the study of automorphisms of Riemann surfaces. Let $\alpha, \beta$, and $\lambda$ be

(4) Using other representations and other finite groups of $W_{0}$, W. T. Kiley has shown the following:

If $g \equiv 1(\bmod 4)$ then $N(g) \geqq 8(g+3)$.

If $g \equiv 5(\bmod 12)$ then $N(g) \geqq 8(g+7)$.

If $g=33(\bmod 48)$ then $N(g) \geqq 8(g+15)$.

(5) If one lifts just the cyclic group of order $2 g+2$, one gets an abelian group of order $4 g+4$, a number which is the upper bound for abelian groups of automorphisms. Also it is possible to construct nonhyperelliptic surfaces admitting groups of order $8 g+8$.

$\left.{ }^{(}{ }^{6}\right)$ The author wishes to express his thanks to Professor H. N. Ward for valuable discussions concerning the material of this section. 
positive integers. An $(\alpha, \beta, \lambda)$ group will be a finite group generated by two elements $a$ and $b$ (of orders $\alpha$ and $\beta$ respectively) whose product has order $\lambda$. We shall also use the notation $\left\langle a_{1}, a_{2}, \ldots\right\rangle$ to denote the group generated by the elements $a_{1}, a_{2}, \ldots$.

The following results may seem somewhat tedious to a reader unfamiliar with the subject of this paper. It is recommended that such a reader omit the remainder of this section on the first reading. The relevence of Lemmas 5, 6, and 7 is more apparent after $\$ 4$ is read.

Let $G$ be a $(2, \beta, \lambda)$ group generated by $a$ and $b$ where $a^{2}=b^{\beta}=e$. Let $c=a b$ and $d=b a$. Then $c^{\lambda}=d^{\lambda}=e$. Assume that the order of $G$ is $\mu \lambda$. We will almost always assume that $\mu$ is even and that $\beta$ divides $\mu$. Our interest is in families of $(2, \beta, \lambda)$ groups where $\beta$ and $\mu$ are fixed and $\lambda$ varies. Consequently, we consider the large cyclic subgroup $\langle c\rangle$. We assert that $\langle c\rangle \cap\langle d\rangle$ is the largest subgroup of $\langle c\rangle$ normal in $G$. Since the order of $a$ is 2 we see that $a c a=d$ and $a d a=c$ so that $a$ and $c$ normalize $\langle c\rangle \cap\langle d\rangle$. Since $\langle a, c\rangle=G,\langle c\rangle \cap\langle d\rangle$ is normal in $G$. Also $\langle d\rangle$ is a subgroup of $G$ conjugate to $\langle c\rangle$, so any subgroup of $\langle c\rangle$, normal in $G$ is in $\langle c\rangle \cap\langle d\rangle$. This proves the assertion. Let $\left\langle c^{k}\right\rangle=\langle c\rangle \cap\langle d\rangle$, where, of course, $k$ divides $\lambda$.

Now it is easy to see that $\langle c, d\rangle$ is a normal subgroup of $G$ which centralizes $\left\langle c^{k}\right\rangle=\langle c\rangle \cap\langle d\rangle$. Also from the fact that $\langle c, d\rangle=\left\langle b^{2}, c\right\rangle$ we see that $\langle c, d\rangle$ has index 1 or 2 in $G$. We now investigate the consequences of the first possibility, $G=\langle c, d\rangle$.

Lemma 4. Let $G$ be a $(2, \beta, \lambda)$ group of order $\mu \lambda$. Suppose that $\mu$ is even and that $\beta$ divides $\mu$. If $G=\langle c, d\rangle$ then $\lambda \leqq \mu^{2}$.

Proof. If $G=\langle c, d\rangle$ then $\left\langle c^{k}\right\rangle$ is central in $G$. Consider the following diagram of subgroups of $G$ :

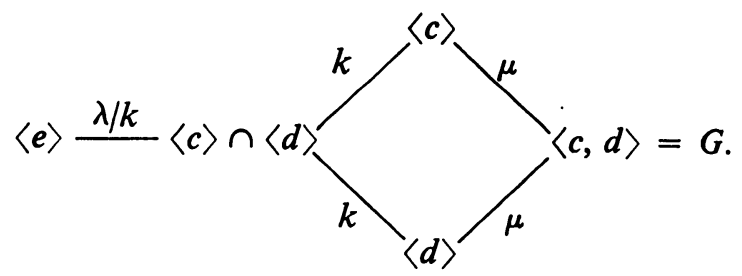

We assert first that $k \leqq \mu$. Let $G^{\prime}$ be the quotient of $G$ by $\langle c\rangle \cap\langle d\rangle$ and let $c^{\prime}$ and $d^{\prime}$ be the elements of $G^{\prime}$ corresponding to $c$ and $d$ in $G$. Then $\left\langle c^{\prime}\right\rangle \cap\left\langle d^{\prime}\right\rangle=\left\langle e^{\prime}\right\rangle$. So the elements $c^{\prime m} d^{\prime n}$ for $m, n=0,1, \ldots, k-1$ are all distinct elements of $G^{\prime}$. Since the order of $G^{\prime}$ is $\mu k$ we have $k^{2} \leqq \mu k$ or $k \leqq \mu$.

Now consider the transfer homomorphism of $G$ into $\left\langle c^{k}\right\rangle$. Since the latter group is assumed to be central, this homomorphism is the map $g \rightarrow g^{k \mu}[9$, Chapter V]. But since 2 and $\beta$ divide $\mu$, this homomorphism takes $a$ and $b$ into the identity; thus, $g \rightarrow g^{k \mu}$ is the zero homomorphism. Consequently, $c^{k \mu}=e$ and $\lambda$ divides $k \mu$. Since $k \leqq \mu$, the proof of the lemma is complete. 
For the next lemma where we let $\beta=3$, we drop the assumption that 2 and $\beta$ divide $\mu$. To obtain the same result we must add the assumption that $G$ is nonabelian.

Lemma $5\left(^{7}\right)$. Let $G$ be a non-abelian $(2,3, \lambda)$ group of order $\mu \lambda$. Then $\lambda \leqq \mu^{2}$.

Proof. In this case $G=\langle c, d\rangle$ since $(2,3)=1$. We now repeat the proof of Lemma 4. To make that proof apply to this lemma we need only show that six divides $k \mu$. Suppose that 2 does not divide $k \mu$. Then $a \in\left\langle c^{k}\right\rangle$ since the index of $\left\langle c^{k}\right\rangle$ in $G$ is $k \mu$. But this implies that $G$ is cyclic, a contradiction. The possibility that 3 does not divide $k \mu$ is handled in exactly the same way. The proof of this lemma is now exactly the same as that of the previous lemma.

From now on we shall assume that $\beta=4$ and consider two cases for $\mu$, namely $\mu=8$ and $\mu=16$. Our assumptions will imply that $\langle c, d\rangle$ has index 2 in $G$. Since $\mu>4,\langle c, d\rangle$ cannot be abelian since $\langle c, d\rangle$ is a $(\lambda, \lambda, 2)$ group. Thus in the following lemmas $G /\left\langle c^{k}\right\rangle$ is a $(2,4, k)$ group of order $k \mu$, for neither $a$ nor $b^{2}$ is in $\left\langle c^{k}\right\rangle$.

Lemma 6. Let $G$ be $a(2,4, \lambda)$ group of order $8 \lambda$, where $\lambda>64$. Then $\lambda \equiv 0(\bmod 3)$ or $\lambda \equiv 0(\bmod 4)$.

Proof. Since $\lambda>64$ ( $=\mu^{2}$ in this case) we have the following diagram of subgroups of $G$ :

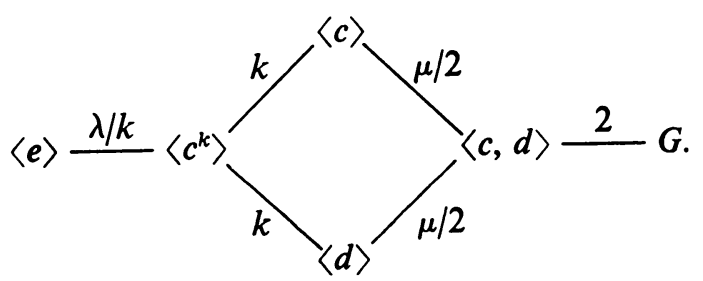

We now consider the possibilities for $k$. As in Lemma 4, we pass to the quotient group $\langle c, d\rangle \mid\left\langle c^{k}\right\rangle$ to conclude that $k \leqq 4$. Suppose that $k=1$. Then $\langle c\rangle=\langle d\rangle$ $=\langle c, d\rangle$ which implies $\mu=2$, a contradiction. If $k=2$ then $\langle c, d\rangle\left\langle c^{k}\right\rangle$ is a $(2,2,2)$ group, that is, the four group. This implies that $\mu=4$, another contradiction. Thus $k=3$ or 4 . Since $k$ divides $\lambda$ the lemma is proved.

LemmA 7. Let $G$ be $a(2,4, \lambda)$ group of order $16 \lambda$ where $\lambda>256$. Then $\lambda \equiv 0(\bmod 4)$ or $\lambda \equiv 0(\bmod 6)$.

Proof. In this case $\mu / 2=8$ and so $k \leqq 8$. As in the previous lemma, $k=1$ or 2 is impossible. If $k=3$ then $G /\left\langle c^{k}\right\rangle$ is a $(2,4,3)$ group, that is $\Sigma_{4}$. In that case $\mu / 2=4$ and $\mu=8$, a contradiction. Thus $4 \leqq k \leqq 8$. To complete the proof of the lemma we show that $k$ is not odd.

(7) A result very similar to Lemma 5 has been proven by Leon Greenberg [1a]. 
From now on we will replace $G$ by $G /\left\langle c^{k}\right\rangle$, using the letters $a, b, c, d$ to refer to elements in this quotient group. Our assumptions imply that $\langle c\rangle$ is not normal in $\langle c, d\rangle$, for if $\langle c\rangle$ is normal in $\langle c, d\rangle$ then $\langle c, d\rangle$ is abelian.

If $k=5$ then Sylow's theorem implies that $\langle c\rangle$ is normal in $\langle c, d\rangle$, a contradiction.

If $k=7$, the fact that $\langle c\rangle$ is not normal implies, again by Sylow's theorem, that $\langle c\rangle$ is its own normalizer in $\langle c, d\rangle$. By a theorem of Burnside, this implies that $\langle c\rangle$ has a normal complement in $\langle c, d\rangle$ which is the 2-Sylow subgroup, $S$, of $\langle c, d\rangle$. Since $S$ is characteristic in $\langle c, d\rangle$, it is normal in $G$. We assert that the nonidentity elements of $S$ are $\left\{b^{2}, c^{-1} b^{2} c, c^{-2} b^{2} c^{2}, \ldots, c^{-6} b^{2} c^{6}\right\}$. These seven elements all have order two. The equality of any two would imply that $b^{2}$ commutes with $c$ and so $\left\langle b^{2}, c\right\rangle=\langle c, d\rangle$ would be abelian. Thus $S$ is the elementary abelian group of order 8. Finally we see that $G / S$, which is isomorphic to the dihedral group of order 14 , act faithfully on $S$ as a group of automorphisms, for $c$ permutes the nonidentity elements cyclicly and $a b^{2} a=c b^{2} c^{-1}=c^{-6} b^{2} c^{6}$.

But $A(S)$ is the simple group of order 168. It has no dihedral group of order 14 as a subgroup since, again, by Sylow's theorem, a subgroup of order 7 must have a normalizer of order 21 . Thus the assumption that $k=7$ leads to this contradiction.

4. The sharpness of the bounds on $N(g)$. We present first in this section some simple lemmas concerning automorphism groups. They are all applications of the Riemann-Hurwitz formula. We always assume that the genus, $g$, of $W$ is greater than 1.

LEMMA 8 (Hurwitz [3]). Suppose $W$ admits a group of automorphisms, $G$, of order $n$ so that $n>4(g-1)$. Then the genus of $W / G$ is zero.

Proof. Let $W_{0}$ be $W / G$ and suppose that $g_{0}$, the genus of $W_{0}$, is positive. The Riemann-Hurwitz formula gives $2 g-2=n\left(2 g_{0}-2\right)+r$. If $g_{0} \geqq 2$, then $2 g-2 \geqq 2 n$ or $n \leqq g-1$. If $g_{0}=1$ then $r \geqq n / 2$ since $g>1$. Consequently, if $g_{0}=1$ we have $2 g-2$ $\geqq n / 2$, or $n \leqq 4(g-1)$. This proves the lemma.

Lemma 9. Let $G$ be a group of automorphisms. Then the prime factors of $o(G)$ occur among the prime factors of $(2 g+1)((g+1) !)$.

Proof. If $S$ is a cyclic group of prime order, $p$, the Riemann-Hurwitz formula gives $2 g-2=p\left(2 g_{0}-2\right)+s(p-1)$, where $s$ is the number of branch points for the covering $W \rightarrow W / S$. If $g_{0} \geqq 2$ then $p \leqq g-1$.

If $g_{0}=1$ then $s$ must be greater than one. For if $s=1$ a representation from the fundamental group of a singly punctured torus (or any singly punctured closed surface) into any abelian group must carry a path "circling" the puncture into the identity since this path is a product of commutators. Thus $s=1$ is impossible. Since $g \geqq 2, s \neq 0$. Consequently $s \geqq 2$ and so $p \leqq g$.

If $g_{0}=0$ we must have $s \geqq 3$ since $g \geqq 2$. For $s=3, p=2 g+1$. For $s \geqq 4$ we have $p \leqq g+1$. 
LEMMA 10. Let $g_{1}$ be an integer greater than 1 . Let $g-1=p\left(g_{1}-1\right)$ where $p$ is prime. Let $S$ be a cyclic group of order $p$ on $W$. Suppose that $p>2 g_{1}+1$. Then the group $S$ is without fixed points; that is, the covering $W \rightarrow W / S$ is an unbranched covering.

Proof. Applying the Riemann-Hurwitz formula to the covering $W \rightarrow W / S$ we have $2 g-2=p\left(2 g_{0}-2\right)+s(p-1)$, where $g_{0}$ is the genus of $W / S$ and $s$ is the number of branch points. Since $g-1=p\left(g_{1}-1\right)$ we have $(p-1) s=2 p\left(g_{1}-g_{0}\right)$. If $g_{0}=g_{1}$ then $s=0$ and the lemma is proven. If $g_{1}>g_{0}$ then $p-1$ divides $2 g_{1}-2 g_{0}$ and so $p-1 \leqq 2 g_{1}-2 g_{0} \leqq 2 g_{1}$. We then reach the contradiction $p \leqq 2 g_{1}+1$.

LEMMA 11. Let $W$ admit a group of automorphisms $G$. Let $N$ be a normal subgroup of $G$. Then $W / N$ admits a group of automorphisms, $H$, isomorphic to $G / N$ and $(W / N) / H=W / G$.

This is a well-known result and the proof is omitted.

THEOREM 1. Let $g_{1}$ be an integer greater than one. Let $p$ be a prime satisfying

(i) $p>84\left(g_{1}-1\right)$,

(ii) $\left((p-1) / 2,\left(2 g_{1}+1\right)\left(\left(g_{1}+1\right) !\right)\right)=1$.

Let $g-1=p\left(g_{1}-1\right)$. Let $W$ be a surface of genus $g$ admitting a group of automorphisms $G$. If $G$ is big then $p$ does not divide $o(G)$.

Proof. We prove that if $p$ divides $o(G)$ then $o(G) \leqq 8(g-1)$.

Since $o(G) \leqq 84(g-1)$ (i) implies that $o(G)=p k$, where $p>k$. Thus the $p$-Sylow subgroup, $S$, of $G$ is normal. Since $p>2 g_{1}+1$ Lemma 10 insures that the covering $W \rightarrow W / S$ is smooth, and the genus of $W / S$ is $g_{1}$. By Lemma $11, W / S$ admits a group isomorphic to $G / S$ of order $k$. Since every prime factor of $k$ divides $\left(2 g_{1}+1\right)\left(\left(g_{1}+1\right) !\right)$ by Lemma 9 and $\left((p-1) / 2,\left(2 g_{1}+1\right)\left(\left(g_{1}+1\right) !\right)\right)=1$, we see that $(p-1, k)$ is 1 or 2 . Thus $(p-1, o(G))$ is 1 or 2 . The kernel, $H$, of the map $G \rightarrow A(S)$ (Lemma 1) has index in $G$ which divides $o(G)$ and $o(A(S))$. Since $o(A(S))=p-1$ we see that $H$ has index 1 or 2 . We complete the proof by showing that $o(H)$ $\leqq 4(g-1)$.

By Lemma $1, S$ is central in $H$ and so, by Lemma 3, $H$ is the direct product of $S$ and $N$ where $N$ is the normal complement of $S$ in $H$. Consider the covering $W \rightarrow W / H$ factored by $W / N$ and $W / S$.

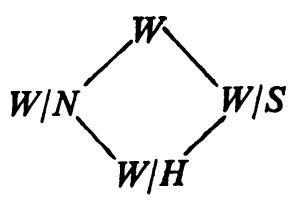

$W \rightarrow W / S$ is a smooth covering. $W / N \rightarrow W / H$ is a $p$ sheeted covering and so must also be smooth. For the covering $W / N \rightarrow W / H$ arises from a cyclic group of order $p$ on $W / N$. If $W / N \rightarrow W / H$ were branched it would lead to an element of order $p$ 
in $H$ with a fixed point, and such an element does not occur since $H \subset G$. Since $p>5, W / H$ admits a nontrivial smooth covering so the genus of $W / H$ is positive. By Lemma $8, o(H) \leqq 4(g-1)$. Q.E.D.

For the following discussion let $g_{1}$ be greater than one, let $p$ satisfy the hypotheses of Theorem 1, let $g=p\left(g_{1}-1\right)$, let the genus of $W$ be $g$, and finally let $G$ be a big group of automorphisms on $W$. Let $o(G)=n$. Theorem 1 assures us that $p$ does not divide $n$. By Lemma 8 , we know that the genus of $W / G$ is zero. Suppose the covering $W \rightarrow W / G$ is branched over $q_{1}, q_{2}, \ldots, q_{s}$, points in $W / G$. How large is the number of such points, $s$ ? By use of the Riemann-Hurwitz formula, Hurwitz [3] proved that if $G$ is big, $s$ is 3 or 4 . Moreover, he showed that the only big groups, for $s=4$, are of order $12(g-1)$, a case ruled out by Theorem 1 . So we know that the branching occurs at precisely three points of $W / G$, the Riemann sphere. Assume that the orders of the branch points are $\alpha, \beta$, and $\lambda$. Then $G$ is an $(\alpha, \beta, \lambda)$ group.

Consequently, we now investigate $(\alpha, \beta, \lambda)$ groups. For such a group the Riemann-Hurwitz formula for the covering $W \rightarrow W / G$ is

$$
2 g-2=n(1-1 / \alpha-1 / \beta-1 / \lambda) \text {. }
$$

LEMma 12. Let $p$ be a prime integer greater than 84. Suppose $G$ is a group of automorphisms of a surface $W$ of genus $g$, where $p$ divides $g-1$. If $p$ does not divide $o(G)$ and $G$ is a big group, then $G$ is a $(2,3, \lambda)$ group or a $(2,4, \lambda)$ group.

Proof. From the preceding discussion we know that $G$ is an $(\alpha, \beta, \lambda)$ group. We may assume that $\alpha \leqq \beta \leqq \lambda$. If $\alpha \geqq 4$ then we see immediately that $n \leqq 8(g-1)$. Next, if $\alpha=3$ and $\beta \geqq 5$, or $\alpha=2$ and $\beta \geqq 8$ we again see that $n \leqq 8(g-1)$. The thirty-five cases to consider can be listed as follows:

$$
\begin{aligned}
& (3,3, \lambda) \text { groups where } 4 \leqq \lambda \leqq 11, \\
& (3,4, \lambda) \text { groups where } 4 \leqq \lambda \leqq 6, \\
& (2,5, \lambda) \text { groups where } 5 \leqq \lambda \leqq 19, \\
& (2,6, \lambda) \text { groups where } 6 \leqq \lambda \leqq 11, \\
& (2,7, \lambda) \text { groups where } 7 \leqq \lambda \leqq 9 .
\end{aligned}
$$

Each case gives rise to a group of order $n$ where $n=(A / B)(g-1)$, where $(A, B)=1$ and $A / B>8$.

As an example, consider the $(2,5,11)$ groups. Here $(2 g-2)=n(1-1 / 2-1 / 5-1 / 11)$ or $n=(220 / 23)(g-1)$ or $23 n=220(g-1)$.

Since $p$ divides $g-1$ but not $n, p$ must divide 23 which is impossible since $p>84$. So $G$ cannot be a $(2,5,11)$ group.

In the general situation we have $B n=A(g-1)$. Since $p$ divides $g-1$ but not $n, p$ must divide $B$. But actual investigation of the thirty-five cases shows that the largest prime factor of $B$ is 47 . Since $p>84$ none of these 35 cases can occur. Q.E.D.

Note that the procedure of Lemma 12 does not work in the $(2,4, \lambda)$ and $(2,3, \lambda)$ cases since there are no bounds on $\lambda$ in these cases. 
The next theorem concerns possible big $(2,3, \lambda)$ groups. It turns out that Lemma 5 eliminates them from the competition if $g$ is large enough.

THEOREM 2. Let $G$ be a group of order $n$ on a surface of genus $g$. Let $g-1$ $=p\left(g_{1}-1\right)$ where $p$ is a prime not dividing $n$. If $n>\left(12\left(g_{1}-1\right)\right)^{3}$ then $G$ is not a $(2,3, \lambda)$ group.

Proof. We assume that $G$ is a $(2,3, \lambda)$ group and show that $n \leqq\left(12\left(g_{1}-1\right)\right)^{2}$.

The Riemann-Hurwitz formula applied to the covering $W \rightarrow W / G$ gives

$$
2 g-2=n(1-1 / 2-1 / 3-1 / \lambda)=n(\lambda-6) / 6 \lambda
$$

or

$$
12 \lambda p\left(g_{1}-1\right)=n(\lambda-6) .
$$

Since $p$ does not divide $n, \lambda-6=\tau p$ where $\tau$ is some integer. Thus $n=12 \tau^{-1}\left(g_{1}-1\right) \lambda$.

Since $G$ is non-abelian, Lemma 5 applied to this group yields $n \leqq\left(12 \tau^{-1}\left(g_{1}-1\right)\right)^{3}$. Since $\tau \geqq 1$ the result follows. Q.E.D.

We see that if the genus of $W$ satisfies the requirements of Theorem 1 and if $8(g+1)>\left(12\left(g_{1}-1\right)\right)^{3}$, then any big group on $W$ must be a $(2,4, \lambda)$ group. Since our examples in $\S 2$ are $(2,4, \lambda)$ groups, we certainly do not want to eliminate them. They occur, in fact, in great profusion. We now apply the analysis of Theorem 2 to $(2,4, \lambda)$ groups.

The Riemann-Hurwitz formula gives

$$
2 g-2=n(1 / 4-1 / \lambda)=n(\lambda-4) / 4 \lambda
$$

or

$$
8 \lambda p\left(g_{1}-1\right)=n(\lambda-4) .
$$

Since $p$ does not divide $n$ we have $\lambda-4=\tau p$ or $8 \lambda\left(g_{1}-1\right)=\tau n$. Since $\lambda=p \tau+4$ we have

$$
\begin{aligned}
n & =8 \tau^{-1}(p \tau+4)\left(g_{1}-1\right) \\
& =8 \tau^{-1}\left(g_{1}-1\right) \lambda \\
& =8\left(g-1+4 \tau^{-1}\left(g_{1}-1\right)\right) .
\end{aligned}
$$

Thus $\tau$ must divide $8\left(g_{1}-1\right)$ since $\lambda$ divides $n$. However, to obtain big groups we must have $4 \tau^{-1}\left(g_{1}-1\right) \geqq 2$. Thus the possible values of $\tau$ which are of interest are those between one and $2\left(g_{1}-1\right)$.

We must now consider more specific values of $g_{1}$. First we let $g_{1}=2$.

THEOREM 3. Let $p$ be a prime satisfying

(i) $p \equiv 59(\bmod 60)\left({ }^{8}\right)$,

(ii) $p>214$.

If $g=p+1$ then $N(g)=8(g+3)$.

(8) Other possible cases are $p \equiv 23$, or $47(\bmod 60)$. 
Proof. Since 3 divides $g$ we know $N(g) \geqq 8(g+3)$ by $\S 2$. For the opposite inequality we let $G$ be a big group of order $n$ and note that the previous analysis of this section applies with $g_{1}=2$. $p$ satisfies the hypotheses of Theorem 1 and Lemma 12 , and $g$ and $p$ satisfy the hypotheses of Theorem 2 . Thus the largest possible big group must be a $(2,4, \lambda)$ group with $\tau$ equal to 1 . This yields $n=8(g+3)$ and so $N(g) \leqq 8(g+3)$. Q.E.D.

By the Dirichlet theorem on primes in an arithmetic series, there are an infinite number of such primes and so this lower bound is attained infinitely often.

To obtain the $8(g+1)$ lower bound infinitely often by the methods of this paper we will let $g_{1}=3$. Unfortunately, this allows a greater variation on $\tau$ and so the proof is longer. Part of the proof of Theorem 4 is contained in Lemmas 6 and 7.

THEOREM 4. Let $p$ be a prime integer satisfying

(i) $((p-1) / 2,42)=1\left(^{9}\right)$,

(ii) $p>863$.

If $g=2 p+1$ then $N(g)=8(g+1)$.

Proof. Let $W$ be a surface of genus $g$ satisfying the above conditions. Let $G$ be a big group of automorphisms of order $n$. The conditions (i) and (ii) insure that Theorem 1, Lemma 12, and Theorem 2 apply. Thus $G$ is a $(2,4, \lambda)$ group and the possible values of $\tau$ are 1,2 , and 4 . Since $\tau=4$ gives $n=8(g+1)$ we wish to show the cases $\tau=2$ and $\tau=1$ are impossible.

Case 1. $\tau=2$. In this case $n=8(g+3), \lambda=2 p+4, \lambda=g+3$ and so $n=8 \lambda$. Since $p$ is odd, $\lambda \equiv 2(\bmod 4)$. Since $\lambda=2(p-1)+6$, the assumption $\lambda \equiv 0(\bmod 3)$ implies that 3 divides $p-1$. This contradicts (i). Now an appeal to Lemma 6 shows that this case is impossible.

Case 2. $\tau=1$. Here $n=8(g+7), \lambda=p+4$ and $n=16 \lambda$. Since $p$ is an odd prime, $\lambda$ must be odd. An appeal to Lemma 7 shows that this case is impossible.

The proof of the theorem is complete.

Again, by the Dirichlet theorem there are an infinite number of primes satisfying (i) and (ii).

\section{REFERENCES}

1. L. R. Ford, Automorphic functions, Chelsea, New York, 1951.

1a. L. Greenberg, Note on normal subgroups of the modular group, Proc. Amer. Math. Soc. 17 (1966), 1195-1198.

2. I. N. Herstein, Topics in algebra, Blaisdell, New York, 1964.

3. A. Hurwitz, Uber algebraische Gebilde mit eindeutigen Transformationen in sich, Math. Ann. 41 (1893), 403-442.

4. A. M. Macbeath, On a theorem of Hurwitz, Proc. Glasgow Math. Assoc. 5 (1961), 90-96.

5. - Proceedings of the Summer School in Geometry and Topology, Queens College, Dundee, 1961.

$\left({ }^{9}\right)$ The possible cases are $p \equiv 11,23,47,59,83(\bmod 84)$. 
6. C. Maclachlan, Groups of automorphisms of compact Riemann surfaces, Thesis, Univ. of Birmingham, England, 1966.

7. H. Röhrl, Unbounded coverings of Riemann surfaces and extensions of rings of meromorphic functions, Trans. Amer. Math. Soc. 107 (1963), 320-341.

8. H. Seifert and W. Threlfall, Lehrbuch der Topologie, Chelsea, New York, 1945.

9. H. Zassenhaus, The theory of groups, Chelsea, New York, 1949.

BROWN UNIVERSITY,

Providence, Rhode Island 